\title{
Damping of non-isothermal hot coronal loops oscillations
}

\author{
M. Luna-Cardozo ${ }^{1}$, R. Erdélyi ${ }^{1}$ and César A. Mendoza-Briceño ${ }^{2}$ \\ ${ }^{1} \mathrm{SP}^{2} \mathrm{RC}$, Department of Applied Mathematics, University of Sheffield, Sheffield S3 7RH, UK \\ emails: m. Iuna; robertus@sheffield.ac.uk \\ ${ }^{2}$ Centro de Física Fundamental CFF, Facultad de Ciencias, Universidad de Los Andes, La \\ Hechicera, Mérida 5101, Venezuela, email: cesar@ula.ve
}

\begin{abstract}
Here we investigate longitudinal waves in non-isothermal hot ( $T \geqslant 5.0 \mathrm{MK})$ coronal loops. Motivated by SOHO SUMER and Yohkoh SXT observations and taking into account gravitational stratification, thermal conduction, compressive viscosity, radiative cooling, and heating, the governing equations of $1 \mathrm{D}$ hydrodynamics is solved numerically for standing wave oscillations along a magnetic field line. A semicircular shape is chosen to represent a coronal loop. It was found that the decay time of standing waves decreases with the increase of the initial temperature and the periods of oscillations are affected by the different initial velocities and loop lengths studied by the numerical experiments. The predicted decay times are within the range of values inferred from Doppler-shift oscillations observed by SUMER in hot coronal loops.
\end{abstract}

Keywords. waves, hydrodynamics, Sun: Corona, Sun: oscillations

\section{Introduction}

Recent observations by high-resolution imaging space telescopes and spectrometers have revealed a variety of coronal oscillation modes. Such oscillations are important because of their potential for the diagnostics of solar atmospheric magnetic structures (magnetic field strength, gas density, etc.) through solar magneto-seismology (Erdélyi 2006a,b). In particular, applications to the corona, using the methods of coronal seismology (proposed by Roberts et al. 1984), help us understanding the heating of the corona. For the most recent review on the topic see e.g. Banerjee et al. (2007). Standing longitudinal slow magnetoacoustic waves have been detected in hot $(T \geqslant 6 \mathrm{MK})$ loops using the SUMER spectrometer on board the SOHO satellite (Kliem et al. 2002; Wang et al. 2002, 2003). These oscillations are excited impulsively, as evidenced by the presence of large initial Doppler shifts and impulsive profiles of intensity and line width. However, unlike the transverse loop oscillations observed by TRACE, the SUMER hot loop oscillations are usually not associated with large flares. They are believed to be excited in the lower parts of the atmosphere near the footpoints (for more details on the excitation see e.g. Taroyan et al. 2007).

Ofman \& Wang (2002) found that thermal conduction is the primary dissipation mechanism of the observed slow waves in hot coronal loops, and Mendoza-Briceño et al. (2004) showed that the inclusion of gravitational stratification results in a further 1020 percent reduction of the damping time. Moreover, Taroyan et al. (2005) considered the additional effect of temperature inhomogeneities on the dissipation of standing slow waves, including thermal conduction and optically thin radiative losses. They found the decay time is proportional to the wave period and the oscillations are rapidly damped mainly due to thermal conduction as in the isothermal loops. 

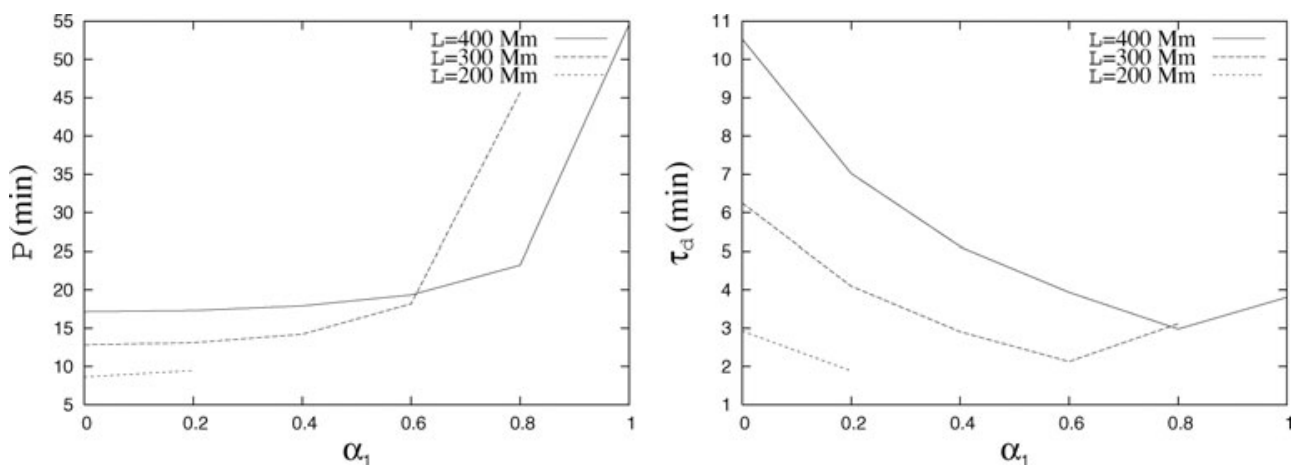

Figure 1. Wave period $P$ (left panel) and decay time $\tau_{d}$ (right panel) as functions of $\alpha_{1}$ parameter, as obtained from the simulations of stratified loops of initial velocity amplitude of $v_{0}=87 \mathrm{~km} / \mathrm{s}$ and $T_{0}=10 \mathrm{MK}$ varying the length from 200 to $400 \mathrm{Mm}$.

Sigalotti et al. (2007) considered the dissipation of standing slow MHD waves in isothermal, hot $(T>6 \mathrm{MK})$ coronal loops, including the effects of gravitational stratification, thermal conduction, compressive viscosity, radiative cooling and heating. They found the decay times are determined mainly by compressive viscosity. Under only thermal conduction, stratification leads to an additional increase of the wave period compared to the homogeneous case, and nonlinearlity, significantly reduced the decay time.

In this paper we reconsider the dissipation of standing slow MHD waves including gravitational stratification, thermal conduction, compressive viscosity, heating, and optically thin radiation losses in non-isothermal hot $(T \geqslant 5 \mathrm{MK})$ coronal loops, solving numerically the (M)HD equations using a 1D, finite-difference code based on a temporally and spatially second order accurate, semi-implicit, Lagrangian solver (Sigalotti \& MendozaBriceño (2003)). In particular, the effects of different initial temperature profiles with maximum temperature at the loop apex up to $20 \mathrm{MK}$ is investigated.

\section{Model and Governing Equations}

Since the plasma dynamics in a coronal loop is dominated by the magnetic field, a usual assumption is made, i.e. the plasma motion takes place primarily along the magnetic field lines, which in turn determines the loop geometry. The energy conservation reads as

$$
\rho \frac{d T}{d t}=-\frac{\bar{\mu}(\gamma-1)}{\mathcal{R}_{g}}\left[p \frac{\partial v}{\partial s}+\rho^{2} Q(T)-H_{0}-\frac{\partial}{\partial s}\left(\kappa \frac{\partial T}{\partial s}\right)-\frac{4}{3} \eta\left(\frac{\partial v}{\partial s}\right)^{2}\right]
$$

where $t$ is time, $s$ denotes the position along a loop of constant cross-section, $\rho$ is the plasma mass density, $v$ is the fluid velocity, $T$ is the plasma temperature, $p$ is the gas pressure, $Q(T)=\chi T^{\alpha}$ is the optically thin radiation-loss function with $\chi$ and $\alpha$ the Hildner's (1974) cooling coefficient and exponent respectively, $H_{0}$ is the constant coronal heating function, $\gamma(=5 / 3)$ is the ratio of specific heats, $\bar{\mu}$ is the mean molecular weight, $\kappa=10^{-6} T^{5 / 2}$ ergs $\mathrm{cm}^{-1} \mathrm{~s}^{-1} \mathrm{~K}^{-1}$ is the coefficient of thermal conductivity parallel to the magnetic field, and $\eta$ is the coefficient of compressive viscosity (Braginskii 1965, Erdélyi \& Goossens 1995). Equation (2.1) together with the continuity and momentum equations are closed by assuming $p=\mathcal{R}_{g} \rho T / \bar{\mu}$, where $\mathcal{R}_{g}$ is the gas constant.

We consider a stratified loop with non-uniform temperature defined as

$$
T(s)=T_{0}\left(1+\alpha_{1} \sin \left(\frac{\pi s}{L}\right)\right),
$$



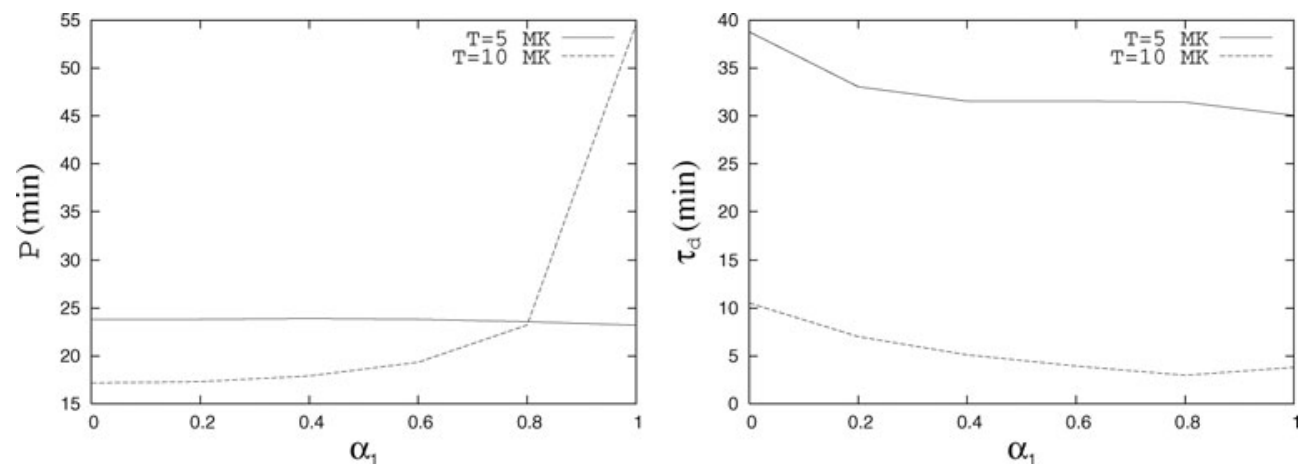

Figure 2. Same as Fig. 1, but obtained from the simulations of stratified loops of length $L=400 \mathrm{Mm}$ and with velocity amplitude $v_{0}=87 \mathrm{~km} / \mathrm{s}$, for the cases of $T_{0}=5$ and $10 \mathrm{MK}$.
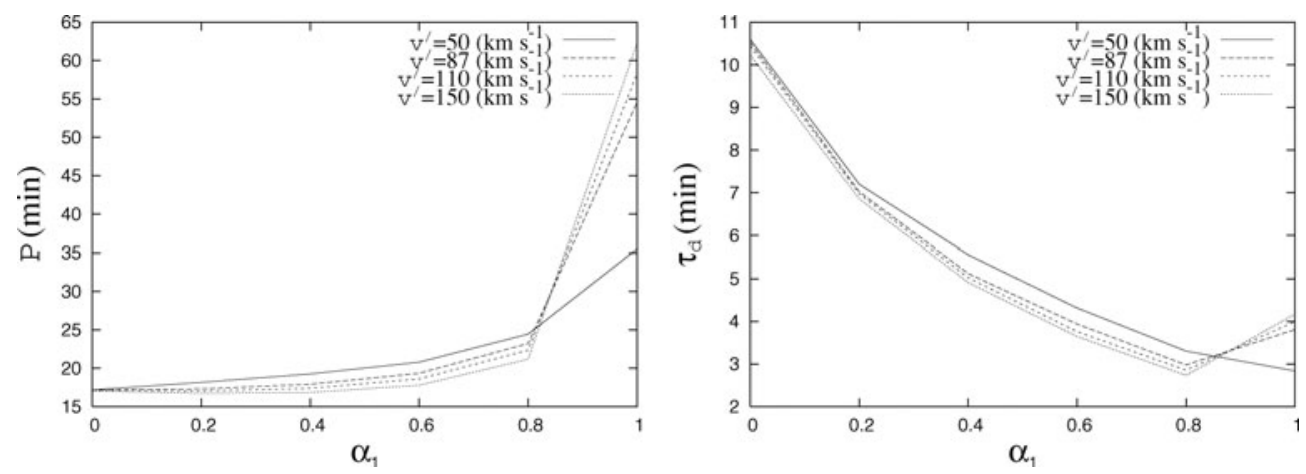

Figure 3. Same as Fig. 1, but obtained from the simulations of stratified loops of length $L=400$ $\mathrm{Mm}$ and $T_{0}=10 \mathrm{MK}$, for a series of initial velocity amplitude varying from 50 to $150 \mathrm{~km} / \mathrm{s}$.

where $T_{0}$ is the loop footpoint temperature and $\alpha_{1} \epsilon[0,1]$ is a dimensionless parameter, controlling the maximum temperature at the apex of the loop.

\section{Results and Discussion}

The set of governing equations are solved numerically. The hydrodynamical variables $\rho, v$, and $T$ obtained from the Lagrangian step are remapped onto an Eulerian mesh, which is reset after each timestep so that the adaptive nature of the Lagrangian solver is preserved. A standing wave problem can be modelled by setting $v=0$ at both ends of the loop along with flow-through boundary conditions for the rest of the variables.

A standing slow wave is modelled by the initial velocity dependence on position of the form $v(s, 0)=v_{0} \sin (\pi s k / L)$, where $v_{0}$ is the amplitude of the wave at $t=0$ and $k$ is the mode number taken to be unity.

The decay time of the velocity perturbation defined by $\tau_{d}=P / \ln \left(v_{0} / v_{1}\right)$, is plotted as function of $\alpha_{1}$ in the right panel of Figs. 1, 2 and 3. In the above relation, $P$ denotes the first complete period of oscillation (left panel of Figs. 1, 2 and 3), while $v_{0}$ and $v_{1}$ are, respectively, the velocity amplitudes at the beginning and after completion of the first cycle of oscillation. These plots are derived from numerical simulations of stratified loops keeping two parameters and varying the remaing one; the parameters are the initial velocity, initial temperature and the loop length. In general, the period of oscillation of standing waves increases and the damping time decreases when $\alpha_{1}$ parameter rises. 
The period and decay time both increase with the loop length when $\alpha_{1} \ll 1$ and decrease when $\alpha_{1} \approx 1$ (very hot loop with $T_{\text {apex }}=20 \mathrm{MK}$ ). The decay time decreases when the initial footpoint temperature $T_{0}$ increases. The period of standing modes decreases with $T_{0}$ for $\alpha_{1} \leqslant 0.8$ and increases for $\alpha_{1}>0.8$. When the initial temperature and length are maintained at $T_{0}=10 \mathrm{MK}$ and $L=400 \mathrm{Mm}$ the period and damping time both decrease with the initial velocity amplitude $v_{0}$ for $\alpha_{1} \leqslant 0.8$ and increase for $\alpha_{1}>0.8$ (see Table 1.).

These predicted decay times are within the range of Doppler-shifts inferred from oscillations observed by SUMER in hot coronal loops (Wang et al. 2003). However, there are not yet enough observations about how the damping time scales with apex temperature.

Table 1. Summary of variation of the period and decay time with the length, initial temperature and initial velocity increase

\begin{tabular}{c|c|c}
\hline & Period $(P)$ & Decay time $\left(\tau_{d}\right)$ \\
\hline Lenght $(L)$ & $\begin{array}{c}\text { increases for } \alpha_{1} \ll 1 \\
\text { and decreases for } \alpha_{1} \approx 1\end{array}$ & $\begin{array}{c}\text { increases for } \alpha_{1} \ll 1 \\
\text { and decreases for } \alpha_{1} \approx 1\end{array}$ \\
\hline Temperature $(T)$ & $\begin{array}{c}\text { decreases for } \alpha_{1} \leqslant 0.8 \\
\text { and increases for } \alpha_{1}>0.8\end{array}$ & decreases for $0<\alpha_{1}<1$ \\
\hline Velocity $\left(v_{0}\right)$ & $\begin{array}{c}\text { decreases for } \alpha_{1} \leqslant 0.8 \\
\text { and increases for } \alpha_{1}>0.8\end{array}$ & $\begin{array}{c}\text { decreases for } \alpha_{1} \leqslant 0.8 \\
\text { and increases for } \alpha_{1}>0.8\end{array}$ \\
\hline
\end{tabular}

\section{Acknowledgements}

$\mathrm{RE}$ is grateful for an IAU travel grant, acknowledges M. Kéray for patient encouragement, and, is also grateful to NSF, Hungary (OTKA, Ref. No. K67746). ML-C is grateful to SU providing an ORS fellowship, and to the IAU for travel support received. C.A.M-B. is grateful to the CDCHT of the Universidad de los Andes for financial support (C-1367-06-05-B).

\section{References}

Banerjee, D., Erdélyi, R., Oliver, R. \& O'Shea, E. 2007, Sol. Phys., 246, 3

Braginskii, S. I. 1965, Rev. Plasma Phys., 1, 205

Erdélyi, R. 2006a, Phil. Trans. Roy. Soc. A, 364, 351

Erdélyi, R. 2006b, in: K. Fletcher (ed.) \& M. Thompson (sci. ed.), SOHO 18/GONG 2006/HELAS I, Beyond the spherical Sun, ESA-SP, 624, 15.1

Erdélyi, R. \& Goossens, M. 1995, A\&A, 294, 575

Hildner, E. 1974, Sol. Phys., 35, 123

Kliem, B., Dammasch, I. E., Curdt, W. \& Wilhelm, K. 2002, ApJ, 568, L61

Mendoza-Briceño, C. A., Erdélyi, R. \& Sigalotti, L. Di G. 2004, ApJ, 605, 493

Ofman, L. \& Wang, T. 2002, ApJ, 580, L85

Roberts, B., Edwin, P. M., \& Benz, A. O. 1984, ApJ, 279, 857

Sigalotti, L. Di G., Mendoza-Briceño, C. A. \& Luna-Cardozo M. 2007, Sol. Phys., in press.

Sigalotti, L. Di G. \& Mendoza-Briceño, C. A. 2003, A\& A, 397, 1083

Taroyan, Y., Erdélyi, R., Doyle, J. G., \& Bradshaw, S. J. 2005, A\&A, 438, 713

Taroyan, Y., Erdélyi, R., Wang, T. J. \& Bradshaw, S. J. 2007 ApJ 659, L173

Wang, T. J., Solanki, S. K., Curdt, W., Innes, D. E., \& Dammasch, I. E. 2002, ApJ, 574, L101

Wang, T. J., Solanki, S. K., Innes, D. E., Curdt, W. \& Marsch E. 2003, A\&A, 402, L17 